\title{
Survival and quality of life in incident systemic sclerosis-related pulmonary arterial hypertension
}

Kathleen Morrisroe ${ }^{1,2}$, Wendy Stevens ${ }^{2}$, Molla Huq ${ }^{1,2}$, David Prior ${ }^{1}$, Jo Sahhar ${ }^{3}$, Gene-Siew Ngian $^{3}$,

David Celermajer ${ }^{4}$, Jane Zochling ${ }^{5}$, Susanna Proudman ${ }^{6,7}$, Mandana Nikpour ${ }^{1,2^{*}}$ and the Australian Scleroderma Interest Group (ASIG)

\begin{abstract}
Background: Pulmonary arterial hypertension (PAH) is a leading cause of mortality in systemic sclerosis (SSc). We sought to determine survival, predictors of mortality, and health-related quality of life (HRQoL) related to PAH in a large SSC cohort with PAH.

Methods: We studied consecutive SSc patients with newly diagnosed (incident) World Health Organization (WHO) Group 1 PAH enrolled in a prospective cohort between 2009 and 2015. Survival methods were used to determine age and sex-adjusted standardised mortality ratio (SMR) and years of life lost (YLL), and to identify predictors of mortality. HRQoL was measured using the Short form 36 (SF-36) instrument.

Results: Among 132 SSc-PAH patients (112 female (85\%); mean age $62 \pm 11$ years), 60 (45.5\%) died, with a median $( \pm I Q R)$ survival time from PAH diagnosis of 4.0 (2.2-6.2) years. Median ( \pm IQR) follow up from study enrolment was 3.8 (1.6-5.8) years. The SMR for patients with SSc-PAH was 5.8 (95\% Cl 4.3-7.8), with YLL of 15.2 years ( $95 \%$ Cl 12.3-18.1). Combination PAH therapy had a survival advantage $(p<0.001)$ compared with monotherapy, as did anticoagulation compared with no anticoagulation $(p<0.003)$. Furthermore, combination PAH therapy together with anticoagulation had a survival benefit compared with monotherapy with or without anticoagulation and combination therapy without anticoagulation (hazard ratio $0.28,95 \% \mathrm{Cl} 0.1-0.7$ ). Older age at PAH diagnosis $(p=0.03$ ), mild co-existent interstitial lung disease (ILD) $(p=0.01)$, worse WHO functional class $(p=0.03)$ and higher mean pulmonary arterial pressure at PAH diagnosis $(p=0.001)$, and digital ulcers ( $p=0.01$ ) were independent predictors of mortality.
\end{abstract}

Conclusions: Despite the significant benefits conferred by advanced PAH therapies suggested in this study, the median survival in SSC PAH remains short at only 4 years.

\section{Background}

Systemic sclerosis (SSc) is a multisystem autoimmune disease, which occurs worldwide with a prevalence ranging from $7 /$ million to $489 /$ million and an incidence ranging from $0.6 /$ million/year to $122 /$ million/year [1]. SSc is characterized by vasculopathy and excessive collagen production, leading to skin and internal organ fibrosis. As there are no effective disease-modifying agents or

\footnotetext{
* Correspondence: m.nikpour@unimelb.edu.au

'Department of Medicine, The University of Melbourne at St Vincent's Hospital, 41 Victoria Parade, Fitzroy, 3065 Melbourne, Victoria, Australia ${ }^{2}$ Department of Rheumatology St Vincent's Hospital, 41 Victoria Parade, Fitzroy, 3065 Melbourne, Victoria, Australia

Full list of author information is available at the end of the article
}

cure, there is substantial morbidity and mortality in this disease.

Despite an improvement over the last three decades, morbidity and mortality in SSc remain high. This is highlighted in a recent large study showing an age and sex adjusted standardised mortality ratio (SMR) of 4.06 for newly diagnosed SSc patients, with 22.4 and 26.0 years of life lost (YLL) in women and men, respectively [2]. Cardiorespiratory manifestations, in particular pulmonary arterial hypertension (PAH), are the leading cause of SSc-related death [3].

$\mathrm{PAH}$ occurs with a prevalence of $8-15 \%$ in SSc patients $[4,5]$. It is characterised by abnormal vascular 
proliferation and remodelling, vasoconstriction and thrombosis of the pulmonary vasculature, leading to elevated pulmonary vascular resistance (PVR), ultimately resulting in right heart failure and death [6]. $\mathrm{PAH}$ is often asymptomatic in the early phases. Once symptomatic, the average life expectancy without treatment has been 2-3 years [6]. Consequently, annual screening with algorithms incorporating transthoracic echocardiogram (TTE) and pulmonary function tests (PFTs) is recommenced [7].

Historically, treatment options for patients with SScPAH are limited [6]. However, in the past decade, with the introduction of new advanced pulmonary vasodilator therapies used as monotherapy or combination therapy, improvement in symptoms, function and survival has been demonstrated $[8,9]$. Currently, there are seven $\mathrm{PAH}$-specific therapeutic agents with regulatory approval available for use in Australia. These agents target the prostacyclin pathway (epoprostenol and iloprost), nitric oxide pathway (sildenafil and tadalafil) or the endothelin pathway (ambrisentan, macitentan and bosentan). Although not available for use in Australia, Riociguat is available in other countries. In Australia, the Pharmaceutical Benefit Scheme (PBS) subsidises monotherapy with one of these agents if prescribed by a physician in a government-designated PAH treatment centre. Once on therapy, patients must demonstrate stability or improvement relative to baseline parameters on two tests (6 minute walk distance (6MWD), TTE or repeat right heart catheterization (RHC)). The $\mathrm{PAH}$-specific therapy can be changed if the patient fails to maintain stability on the aforementioned tests. Combined PAHspecific therapy, using two or more drugs with different modes of action can only occur by compassionate access through hospital pharmacies or the manufacturers, or at patients' own expense. Anticoagulation in the treatment of PAH is a contentious issue in SSc, with some studies showing a survival benefit in patients with idiopathic PAH (iPAH) and connective tissue disease (CTD)-associated PAH $[10,11]$ and others not showing a survival benefit [12]. Furthermore anticoagulation in SSc is not without risk.

Despite an improvement in survival with these therapies, survival in SSc-PAH remains well below that of iPAH and CTD-PAH [13] with one-year, two-year and three-year survival of $90 \%, 78 \%$ and $56 \%$, respectively compared with one-year, three-year and five-year survival in idiopathic $\mathrm{PAH}$ of $92 \%, 75 \%$ and $66 \%$, respectively $[13,14]$. Survival in incident SSc-PAH may be below this as these figures are derived from incident and prevalent SSc-PAH cohort data, introducing a survival bias. Not only does SSc-PAH affect patient survival, it also has a significant impact on patients' functional capacity and health-related quality of life (HRQoL) $[15,16]$.
We sought to determine survival and HRQoL related to incident SSc-PAH in a large cohort of Australian SSc patients, and to identify predictors of mortality.

\section{Methods}

\section{Patient cohort}

All patients fulfilled either the American College of Rheumatology criteria for SSc or Leroy and Medsger criteria for SSc $[17,18]$. Patients included in this analysis were from the Australian Scleroderma Cohort Study (ASCS). The ASCS is a prospective multi-centre study of risk and prognostic factors for cardiopulmonary outcomes in SSc. The ASCS compromises 13 Australian centres and has been approved by the human research ethics committee of each of the participating hospitals (St. Vincent's Hospital, Melbourne Royal Adelaide Hospital, Monash Medical Centre, Royal Perth Hospital, The Queen Elizabeth Hospital, Sunshine Coast Rheumatology, Prince Charles Hospital, John Hunter Hospital, Royal North Shore Hospital, Royal Prince Alfred Hospital, St George Hospital, Canberra Rheumatology and the University of Tasmania). All patients provide written informed consent at recruitment.

\section{Inclusion and exclusion criteria}

All patients were screened annually for PAH with PFTs and TTE. Any patient identified as at high risk of developing $\mathrm{PAH}$, defined as systolic pulmonary arterial pressure $\left(\mathrm{sPAP}_{\mathrm{TTE}}\right)$ of at least $50 \mathrm{mmHg}$ and/or diffusing lung capacity for carbon monoxide (DLCO) $<50 \%$ predicted with forced vital capacity (FVC) $>85 \%$ predicted, without adequate explanation on high-resolution computer tomography (HRCT) of the chest or ventilationperfusion $(\mathrm{V} / \mathrm{Q})$ scan of lung or both, underwent RHC.

We included all consecutive adult (age $>18$ years) SSc patients from the ASCS between June 2009 and June 2015, who were diagnosed with World Health Organization (WHO) Group 1 PAH on RHC (mean pulmonary arterial pressure (mPAP) of at least $25 \mathrm{mmHg}$ and pulmonary arterial wedge pressure (PAWP) $<15 \mathrm{mmHg}$ ) [19].

Patients were excluded if they had WHO Group 2 or 3 pulmonary hypertension or Group $1 \mathrm{PAH}$ but with coexisting ILD with FVC $<60 \%$ and abnormal HRCT of the chest. V/Q scanning was used to exclude pulmonary hypertension due to chronic thromboembolism.

\section{Data collection}

Patient demographics, clinical variables and cardiac and pulmonary assessments were obtained from the ASCS database. All physical examination and investigation data were collected within one month of the first RHC, before starting pulmonary vasodilator therapy. Clinical manifestations and autoantibody status were defined as 
present, if ever present from SSc diagnosis. Scleroderma disease onset and disease duration were defined from the date of onset of the first non-Raynaud manifestation. Autoantibodies measured included anti-nuclear antibodies (ANA), antibodies to extractable nuclear antigens (ENA), anti-RNA polymerase III antibodies, anti-Scl-70 antibody and antiphospholipid antibodies (APLA). TTE was performed according to standardised procedures only at tertiary centres with expertise. Pulmonary involvement was assessed by PFTs and HRCT.

Patient-reported outcome measures were collected annually, including the SSc-specific health assessment questionnaire (SHAQ) and the Medical Outcomes Study Short Form-36 (SF-36), a functional assessment tool and a health-related quality of life measurement tool, respectively, which are both well-validated for use in SSc [20]. These patient-reported outcomes (PROs) were chosen as they are collected annually for each patient in the ASCS.

Demographics and clinical manifestations were compared between SSc patients who developed and those that did not develop PAH. Furthermore, PRO scores were compared between these two groups.

\section{Outcome variables}

The principal outcome variable was all-cause mortality. The date of death was recorded. Where data were available, the exact cause of death was recorded. Patient status (alive or dead) at the time of censoring (January 2016) was confirmed by checking with the treating physician and verified against hospital records. The secondary outcome variables that we evaluated were the most recent SHAQ score and the physical and mental component scores of the SF-36 (PCS and MCS) following PAH treatment.

\section{PAH therapy and other medications}

All specific PAH therapies (endothelin receptor antagonists (ERA), phosphodiesterase-5-inhibitors (PDE5) and prostacyclin analogues) and their combinations (monotherapy or combination therapy) were prescribed at the discretion of the managing physician(s) and these medications were recorded at each visit. Use of other therapeutic agents such as anticoagulation (including indication, date of initiation and target international normalised ratio (INR) for warfarin, date and reason for cessation of anticoagulation), antiplatelet agents, hydroxychloroquine (HCQ), mycophenolate mofetil (MMF), hormone replacement therapy (HRT) and proton pump inhibitors (PPIs) were also at the discretion of the managing physician(s) and were recorded.

\section{Statistical analysis}

Patient characteristics at baseline are presented as mean \pm standard deviation for continuous variables and as number (percentage) for categorical variables. Allcause mortality was used for analyses because causes of death could not always be confidently ascribed. KaplanMeier (K-M) curves were used to estimate survival in patients with SSc-PAH. One-year, two-year and three-year survival were assessed; date of RHC diagnosis of PAH was considered the baseline from which survival was measured. The log-rank and Wilcoxon tests were used to compare survival curves. The SMR was calculated using the observed deaths in our SSc cohort and the expected deaths in the Australian population, which was sourced from the Australian Bureau of Statistics (ABS). YLL was also calculated based on Australian life expectancy using ABS data.

After testing to ensure proportionality of hazard, Cox proportional hazards regression analyses were used to determine univariable and multivariable predictors of mortality. All variables a with $p$ value $\leq 0.1$ in univariable analysis or variables with clinical face validity were selected for inclusion in multivariable analysis. The results were reported as hazard ratios (HR) with accompanying 95\% confidence intervals (CI). Mixed effect linear regression was used to identify and quantify determinants of the SHAQ score and the PCS and MCS of the SF-36 following PAH treatment. A two-tailed $p$ value $\leq 0.05$ was considered statistically significant. All statistical analyses were performed using STATA 14.0 (StataCorp LP, College Station, TX, USA).

\section{Results}

\section{Patient characteristics}

Of the 1578 SSc patients enrolled in ASCS, 132 patients were diagnosed with incident Group 1 SSc-PAH and included in this study. Patient characteristics by PAH status are summarised in Additional file 1: Table S1. SSc$\mathrm{PAH}$ patient characteristics and haemodynamic measurements are summarised in Table 1. Our SSc-PAH cohort compromised predominantly women (84.9\%) with limited disease subtype (limited cutaneous systemic sclerosis (lcSSc)) (68.9\%) and a mean (IQR) follow-up duration of 3.8 (1.6-5.8) years since ASCS recruitment. At PAH diagnosis, the mean SSc disease duration was $14.1 \pm 11.9$ years, with no difference between disease subtypes $(p=0.40)$. Anti-centromere ANA was the most common autoantibody detected (51.6\%), followed by APLA (30\%). Anti-Scl-70 was infrequent (7.4\%).

Despite annual screening, the majority of patients at PAH diagnosis were in WHO functional class II (17.4\%) or class III (59.9\%) with a mean baseline 6MWD of $326.1( \pm 105.5) \mathrm{m}$. Hemodynamics measured at the time of PAH diagnosis showed moderate PAH with an mPAP of $35.6( \pm 10.4) \mathrm{mmHg}$, mean right atrial pressure (mRAP) of $8.3( \pm 4.3) \mathrm{mmHg}$ and mean cardiac index $(\mathrm{mCI})$ of $3.2( \pm 1.9) \mathrm{L} / \mathrm{min} / \mathrm{m}^{2}$. Mean DLCO at PAH 
Table 1 Characteristics of patients with SSC-PAH

\begin{tabular}{|c|c|}
\hline Characteristic & $\begin{array}{l}\text { Mean }( \pm \mathrm{SD}) \text {, number } \\
\text { (percent) or median (IQR) }\end{array}$ \\
\hline Total number of patients & 132 \\
\hline Female & $112(85 \%)$ \\
\hline Age at PAH diagnosis, years & $62.3( \pm 10.9)$ \\
\hline Disease duration ${ }^{\mathrm{a}}$ at PAH diagnosis, years & $14.1( \pm 11.9)$ \\
\hline \multicolumn{2}{|l|}{ Status at censoring } \\
\hline Alive & $70(53.0 \%)$ \\
\hline Dead & $60(45.5 \%)$ \\
\hline Withdrawn & $1(0.8 \%)$ \\
\hline Unable to contact & $1(0.8 \%)$ \\
\hline \multicolumn{2}{|l|}{ Race } \\
\hline Caucasian & $112(84.9 \%)$ \\
\hline Asian & $6(4.6 \%)$ \\
\hline Aboriginal-Islander & $1(0.8 \%)$ \\
\hline Hispanic & $1(0.8 \%)$ \\
\hline Follow-up duration ${ }^{\text {b }}$, years (median (IQR)) & $3.8(1.6-5.8)$ \\
\hline Survival from PAH diagnosis, years (median (IQR)) & $4.0(2.2-6.2)$ \\
\hline Disease duration ${ }^{\mathrm{a}}$ at PAH diagnosis, years & $14.4 \pm 12.1$ \\
\hline \multicolumn{2}{|l|}{ Disease subtype } \\
\hline Limited & $91(68.9 \%)$ \\
\hline Diffuse & $30(22.7 \%)$ \\
\hline MCTD & $7(5.3 \%)$ \\
\hline \multicolumn{2}{|l|}{ Autoantibody status } \\
\hline Anti-centromere pattern ANA & $63(51.6 \%)$ \\
\hline Antiphospholipid antibodies (>ULN) & $33(30 \%)$ \\
\hline RNA polymerase III positive & $8(11.4 \%)$ \\
\hline Scl 70 positive & $9(7.4 \%)$ \\
\hline \multicolumn{2}{|l|}{ WHO functional class at time of PAH diagnosis } \\
\hline Class 1 & $3(2.3 \%)$ \\
\hline Class ॥ & $23(17.4 \%)$ \\
\hline Class III & 79 (59.9\%) \\
\hline Class IV & $12(9.1 \%)$ \\
\hline Baseline 6MWD, m & $326.13( \pm 105.5)$ \\
\hline Baseline mRAP, mmHg & $8.3( \pm 4.3)$ \\
\hline Baseline mPAP, mmHg & $35.6( \pm 10.4)$ \\
\hline Baseline PAWP, mmHg & $10.5( \pm 3.4)$ \\
\hline Baseline $\mathrm{mCl}, \mathrm{L} / \mathrm{min} / \mathrm{m} 2$ & $3.2( \pm 1.9)$ \\
\hline Baseline PVR, Wood units & $8.7( \pm 3.8)$ \\
\hline Presence of a pericardial effusion at PAH diagnosis & $24(18.2 \%)$ \\
\hline Mean DLCO, \% predicted mL/min $/ \mathrm{mmHg}$ & $46.6( \pm 13.5)$ \\
\hline Mean DLCO $/ \mathrm{AA}, \%$ predicted $\mathrm{mL} / \mathrm{min} / \mathrm{mmHg}$ & $56.7( \pm 20.2)$ \\
\hline \multicolumn{2}{|l|}{ Medical therapy } \\
\hline \multicolumn{2}{|l|}{ Pulmonary vasodilator therapy ${ }^{c}$} \\
\hline Monotherapy & $91(68.9 \%)$ \\
\hline Combination therapy & 41 (31.1\%) \\
\hline Warfarin therapy ${ }^{d}$ & $37(28.5 \%)$ \\
\hline
\end{tabular}

Table 1 Characteristics of patients with SSc-PAH (Continued)

$\begin{array}{ll}\text { Hydroxychloroquine therapy }^{d} & 12(9.1 \%) \\ \text { Antiplatelet agent }^{c} & 48(36.9 \%) \\ \text { Mycophenolate mofetil therapy }^{d} & 7(5.4 \%) \\ \text { Hormone replacement therapy }^{d} & 16(12.3 \%) \\ \text { Proton pump inhibitor }^{d} & 105(80.8 \%) \\ \text { Home oxygen }^{d} & 28(21.5 \%)\end{array}$

Abbreviations: SSC systemic sclerosis, PAH pulmonary arterial hypertension, MCTD mixed connective tissue disease, ANA antinuclear antibody, ULN upper limit of normal, WHO World Health Organization, 6MWD six-minute walk distance, $M R A P$ mean right atrial pressure, $M P A P$ mean pulmonary arterial pressure, PAWP pulmonary artery wedge pressure, PVR peripheral vascular resistance, $\mathrm{mCl}$ mean cardiac index, $D L C O$ diffusing capacity of the lung for carbon monoxide, DLCO/VA DLCO adjusted for alveolar volume ${ }^{a}$ Disease duration from first non-Raynaud manifestation

${ }^{\mathrm{b}}$ Follow-up duration was defined as years from study enrollment

${ }^{\mathrm{C}}$ Monotherapy is treatment with a single PAH-specific therapy. Combination therapy is treatment with more than one specific PAH agent from different classes at one time

${ }^{d}$ Treatment ever following the diagnosis of PAH

diagnosis was $46.6 \%( \pm 13.5)$ predicted, and DLCO corrected for alveolar volume (DLCO/VA) was 56.7\% $( \pm 20.2)$ predicted. A pericardial effusion was present at $\mathrm{PAH}$ diagnosis in $18.2 \%$ of patients.

\section{Specific PAH therapy}

All patients were treated with at least one specific PAH medication. Considering the Australian PBS regulations, in our study, the majority of patients (68.9\%) were treated with monotherapy (including sequential therapy) and $31.1 \%$ with combination therapy (two or more advanced PAH therapies at the same time). Six patients received upfront combination therapy at the time of PAH diagnosis. The remainder of patients (31 patients (26.5\%)) on combination therapy received additional therapy as "add-on" therapy due to functional deterioration. Medications were altered at physician discretion based on failure of the specific PAH therapy or adverse effects.

As monotherapy, bosentan (68.1\%) was the most commonly prescribed drug followed by sildenafil (15.9\%). Other monotherapy prescribed and its frequency included ambrisentan (8.7\%), macitentan (2.9\%) and sitaxentan (before its withdrawal) (2\%). The most common combination was bosentan and sildenafil (49.1\%) followed by bosentan and tadalafil (12.3\%). Supplemental home oxygen was used by $21.5 \%$ of patients.

Patients treated with combination therapy compared with monotherapy had more severe PAH reflected by a higher mPAP $(39.4( \pm 11.9)$ vs. $34.1( \pm 10.4) \mathrm{mmHg}$, $p=0.007)$, mPVR $(6.2( \pm 3.2)$ vs. $4.3( \pm 2.5)$ Wood Units, $p=0.003)$, lower DLCO percent than predicted (41.4 ( \pm 11.8$)$ vs. $49.7( \pm 13.5), p=0.003)$ and the presence of a pericardial effusion $(36.6 \%$ vs. $11.3 \%, p=0.001)$ at $\mathrm{PAH}$ diagnosis. There was also a trend towards more 
digital ulcers $(68.3 \%$ vs. $49.4 \%, \mathrm{p}=0.06)$ at PAH diagnosis in those commenced on combination therapy compared with monotherapy. There was no difference in mRAP $(p=0.37), \mathrm{mCI}(2.7( \pm 0.9)$ vs. $3.5( \pm 2.1) \mathrm{L} / \mathrm{min} / \mathrm{m} 2$, $p=0.21)$, age at PAH diagnosis $(p=0.38)$ or disease subtype $(p=0.47)$ (Additional file 1: Table S2) in combination versus monotherapy.

\section{Anticoagulation and other medical therapies}

In our cohort of SSc-PAH patients, $28.5 \%$ were anticoagulated with warfarin, $36.9 \%$ were on an antiplatelet agent, $80.8 \%$ on a PPI, $12.3 \%$ on HRT, $9.1 \%$ on HCQ and $5.4 \%$ on MMF (for treatment of their skin disease). Nine patients were on both warfarin and aspirin concurrently.

In those who were treated with warfarin, 54.1\% were initiated on warfarin specifically for the treatment of $\mathrm{PAH}$ and $45.9 \%$ were placed on warfarin for another indication following the diagnosis of PAH. Eleven PAH patients had to cease their anticoagulation after their PAH diagnosis due to complications of warfarin therapy including gastrointestinal bleeding (which accounted for $58.3 \%$ of reasons for stopping warfarin) and difficulty monitoring the INR (INR target 1.5-2.5).

Patients on anticoagulation had more severe PAH reflected by higher mPVR $(6.2( \pm 3.6)$ vs. $4.5( \pm 2.5)$ Wood units, $p=0.02)$, lower $\mathrm{mCI}(2.4( \pm 0.7)$ vs. 3.7 $( \pm 1.8) \mathrm{L} / \mathrm{min} / \mathrm{m} 2, p=0.007)$, shorter 6MWD (291.3 $( \pm 100.3)$ vs. $340.2( \pm 104.9) \mathrm{m}, p=0.01)$, lower $\operatorname{mDLCO}(42.3( \pm 12.5)$ vs. $48.6( \pm 13.5) \mathrm{mL} / \mathrm{min} /$ $\mathrm{mmHg}, p=0.05)$ and the presence of a pericardial effusion $(36.1 \%$ vs. $12.9 \%, p=0.003)$ at PAH diagnosis. There was no difference in mRAP $(p=0.19)$, mPAWP $(p=0.99), \mathrm{mDLCO} / \mathrm{VA}(50.0( \pm 21.8)$ vs. $59.4( \pm 19.3) \mathrm{mL} /$ $\mathrm{min} / \mathrm{mmHg}, p=0.21)$, mPAP $(39.5( \pm 14.1)$ vs. $34.5( \pm 9.3)$ $\mathrm{mmHg}$, age at PAH diagnosis $(p=0.88)$, disease subtype $(p=0.85)$ or presence of digital ulcers $(p=0.94)$ (Additional file 1: Table S2) in those who were anticoagulated compared with those who were not.

Of note, $37.5 \%$ of patients (6 patients) with a known history of gastric antral vascular ectasia (GAVE), defined as characteristic vascular lesions seen on endoscopy, but without recent bleeding, were anticoagulated with warfarin, while only $27.2 \%$ of patients (31 patients) with $\mathrm{PAH}$ and no history of GAVE were anticoagulated. This further highlights that many factors, not only GAVE, influence an individual physician's decision to prescribe anticoagulation in this group of patients.

\section{Survival in SSc-PAH}

SSc-PAH had a significant impact on survival $(p<0.001)$ (Fig. 1). Over a median ( \pm IQR) follow-up of 3.8 (1.6$5.8)$ years from study enrolment, 60 (45.5\%) patients died with a median $( \pm$ IQR) survival time from $\mathrm{PAH}$ diagnosis of $4.0(2.2-6.2)$ years. One-year, two-year,

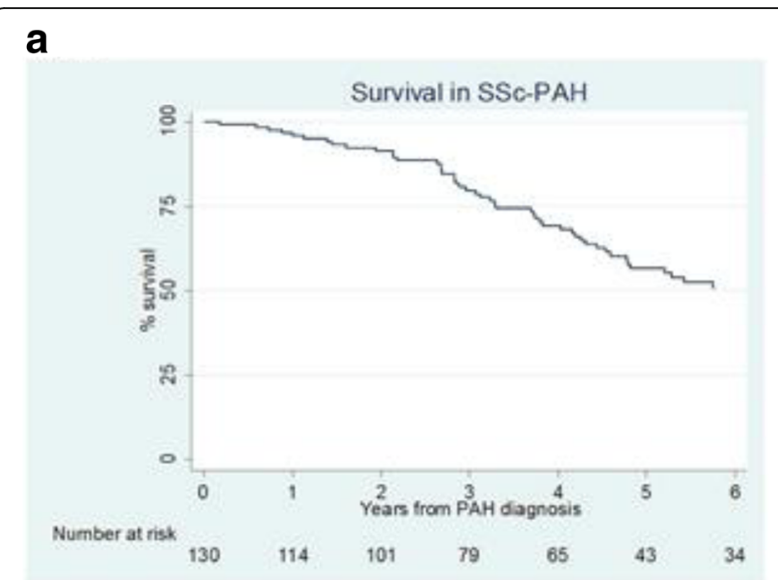

b

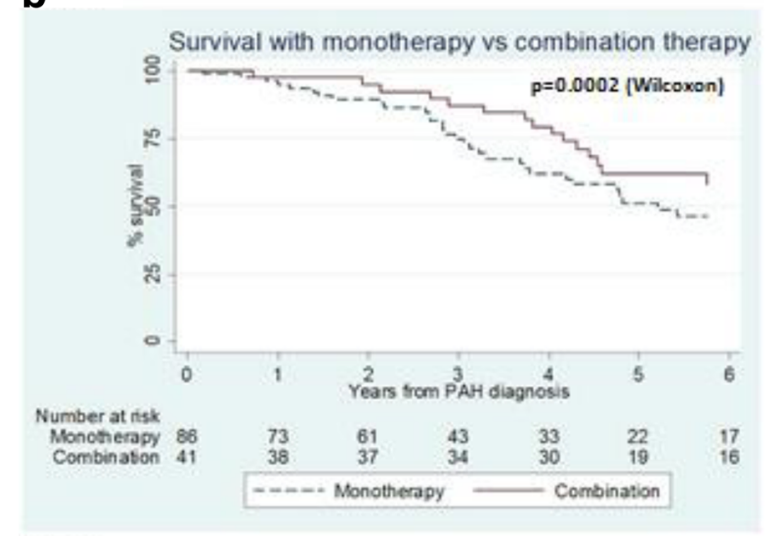

C

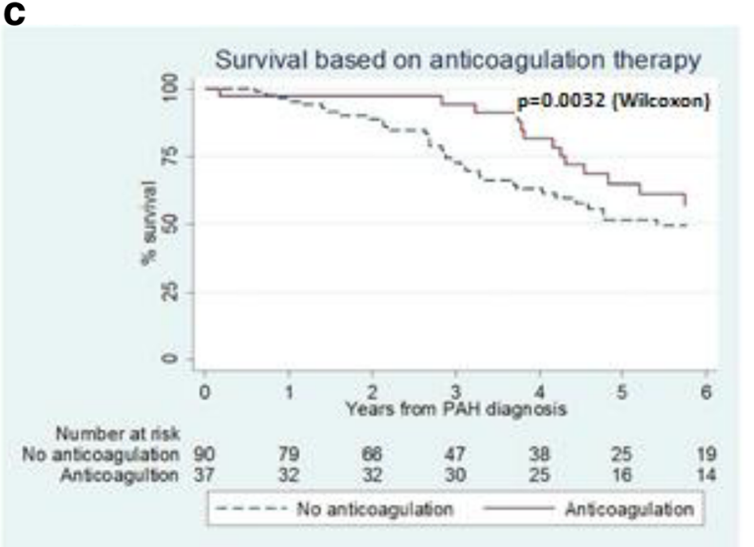

Fig. 1 Survival in systemic sclerosis with pulmonary hypertension (SSC-PAH). a Survival in SSC-PAH. b Survival with monotherapy vs combination therapy. c Survival based on anticoagulation therapy

three-year and five-year survival was 87.8\%, 78.3\%, $61.7 \%$ and $32.2 \%$, respectively. The age and sex adjusted SMR for patients with SSc-PAH compared with mortality in the general population was 5.8 (95\% CI 4.3-7.3). The overall YLL for both male and female patients due to SSc-PAH was 15.2 years (95\% CI 12.3-18.1). Men had higher YLLs than women (17.0 years (95\% CI 7.7-23.0) compared with 15.4 years $(13.8-20.3)$ ). The majority of 
deaths were directly related to PAH (70\%), with PAH being a significant contributor in the remaining causes of death (malignancy (13.3\%), gastrointestinal complication (10\%), renal (3.3\%), and infection (3.3\%)).

In univariable analysis (Additional file 1: Table S3), factors associated with mortality included the presence of calcinosis ever, worse WHO functional class, shorter 6MWD, higher mPAP and MPVR and lower DLCO at PAH diagnosis, home oxygen use and lack of PPI use.

Independent predictors of mortality in SSc-PAH in multivariable hazards regression analysis are summarised in Table 2. To ensure model stability, a desired ratio of independent-to-outcome variables was set at one to ten. Older age at PAH diagnosis (HR 1.1, 95\% CI 1.0-1.1, $p=0.03$ ), presence of mild ILD (HR 2.8, 95\% CI 1.4-5.6, $p=0.01$ ), worse WHO functional class (HR 2.0, 95\% CI 1.1-3.9, $p=0.03$ ), higher mPAP at PAH diagnosis (HR 1.1, 95\% CI 1.0-1.1 mmHg, $p=0.001)$ and presence of digital ulcers ever (HR 3.1, 95\% CI 1.4-7.2, $p=0.01$ ) were predictive of mortality. The 6MWD was not predictive of mortality. Using PAH monotherapy as our reference group, the addition of anticoagulation to monotherapy was associated with a trend towards survival benefit $(p=0.09)$. Additionally, PAH combination therapy (all cases were a combination of a PDE5 inhibitor and an ERA) also showed a trend towards a survival benefit compared with monotherapy alone $(p=0.10)$. Furthermore, combination PAH therapy, together with anticoagulation, provided the most significant survival advantage with a $72 \%$ reduction in mortality compared with pulmonary vasodilator monotherapy alone (HR 0.28, 95\% CI 0.1-0.7, $p=0.01$ ).

Kaplan-Meier survival curves (Fig. 1) depict the survival advantage with combination PAH therapy

Table 2 Independent predictors of mortality in SSc-PAH determined by multivariable Cox proportional hazard regression analysis

\begin{tabular}{|c|c|c|}
\hline Characteristic & $\begin{array}{l}\text { Hazard ratio } \\
(95 \% \mathrm{Cl})\end{array}$ & $P$ value \\
\hline Age at diagnosis of $\mathrm{PAH}$, years & $1.1(1.0-1.1)$ & 0.03 \\
\hline ILD on HRCT (FVC >60\%) & $2.8(1.4-5.6)$ & 0.01 \\
\hline WHO functional class & $2.0(1.1-3.9)$ & 0.03 \\
\hline Pulmonary arterial pressure at $\mathrm{PAH}$ diagnosis, $\mathrm{mmHg}$ & $1.1(1.0-1.1)$ & 0.001 \\
\hline Digital ulcers present ever & $3.1(1.4-7.2)$ & 0.01 \\
\hline \multicolumn{3}{|l|}{ Specific PAH therapies and anticoagulation } \\
\hline Vasodilator monotherapy only & reference & reference \\
\hline Vasodilator monotherapy and anticoagulation & $0.39(0.1-1.2)$ & 0.09 \\
\hline Vasodilator combination therapy only & $0.49(0.2-1.2)$ & 0.10 \\
\hline Vasodilator combination therapy and anticoagulation & $0.28(0.1-0.7)$ & 0.01 \\
\hline \multicolumn{3}{|c|}{$\begin{array}{l}\text { Abbreviations: SSC systemic sclerosis, } P A H \text { pulmonary arterial hypertension, } \\
\text { WHO world health organization, ILD interstitial lung disease, HRCT high-resolution } \\
\text { computer tomography. FVC forced vital capacity, } 6 M W D \text { six-minute walk distance, } \\
\text { mRAP mean right atrial pressure, mPAP mean pulmonary arterial pressure, } \\
\text { HCQ hydroxychloroquine }\end{array}$} \\
\hline
\end{tabular}

compared with monotherapy $(p<0.001)$ and anticoagulation compared with no anticoagulation $(p<0.003)$. Mean time $( \pm S D)$ to death was longer for patients who were anticoagulated than those who were not $(5.4( \pm 2.5)$ vs. $3.5( \pm 2.1), p=0.001$ ) and for those on PAH combination therapy compared to those on monotherapy (5.2 $( \pm 2.8)$ vs. 3.5 ( \pm 1.9 ), $p=0.02$ ). There was no difference in mean time to death in those with and without APLA on anticoagulation $(p=0.68)$ or those with limited versus diffuse disease subtypes $(p=0.56)$.

\section{Patient-reported outcome measures in SSc-PAH}

In relation to physical function, patients with SSc-PAH had significantly lower SHAQ scores indicating significant functional limitation in their daily activities compared with SSc patients without PAH (Table 3). Determinants of a better SHAQ score using mixed

Table 3 Patient-reported outcomes in patients with SSc-PAH compared to patients with SSC without PAH

\begin{tabular}{llll}
\hline Outcomes & PAH & No PAH & $P$ value \\
\hline SHAQ domain $^{\text {a }}$ & & & \\
Patient number & 132 & 1447 & \\
Total score & $3.2 \pm 1.7$ & $3.3 \pm 2.1$ & 0.74 \\
Breathing & $4.9 \pm 2.5$ & $2.3 \pm 2.4$ & $<0.001$ \\
Digital ulcers & $1.9 \pm 2.5$ & $1.4 \pm 2.4$ & 0.02 \\
Intestinal & $2.8 \pm 2.5$ & $2.2 \pm 2.4$ & 0.002 \\
Patient global assessment & $5.1 \pm 2.2$ & $3.6 \pm 2.4$ & $<0.001$ \\
Pain & $3.9 \pm 2.6$ & $3.4 \pm 2.6$ & 0.03 \\
Vascular (RP) & $3.3 \pm 2.5$ & $2.7 \pm 2.5$ & 0.01 \\
SF-36 domain & & & \\
Physical functioning & $35.7 \pm 23.8$ & $57.5 \pm 28.9$ & $<0.001$ \\
Role limitation, physical & $27.2 \pm 39.1$ & $49.2 \pm 43.4$ & $<0.001$ \\
Role limitation, emotional & $55.9 \pm 44.9$ & $67.3 \pm 40.4$ & 0.05 \\
Social functioning & $64.2 \pm 27.7$ & $70.5 \pm 26.9$ & 0.07 \\
Mental health & $66.1 \pm 21.1$ & $68.9 \pm 20.2$ & 0.28 \\
Energy/vitality & $38.6 \pm 22.2$ & $47.1 \pm 24.1$ & 0.01 \\
Bodily pain & $55.3 \pm 28.8$ & $60.7 \pm 27.9$ & 0.10 \\
General health perception & $36.6 \pm 20.5$ & $46.2 \pm 22.7$ & 0.01 \\
Physical component score & $31.7 \pm 8.7$ & $38.9 \pm 11.6$ & $<0.001$ \\
Mental component score & $46.3 \pm 10.7$ & $46.3 \pm 10.4$ & 0.48 \\
\hline
\end{tabular}

Systemic sclerosis (SSc)-specific health assessment questionnaire (SHAQ) and Medical Outcomes Study Short Form-36 (SF-36) values are based on the average of all instrument scores collected annually in the database. For those with pulmonary hypertension $(\mathrm{PAH})$, this includes only those scores that have been collected since PAH diagnosis. For those without PAH this includes all scores within the database for these patients. Raynaud's phenomenon (RP)

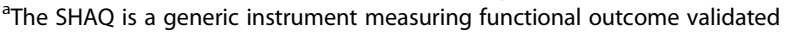
for use in SSc. The score ranges from 0 to 10 , with 0 being no functional limitation and 10 being severe functional limitation

${ }^{\mathrm{b}}$ The SF-36 form is a 36 -item scale that measures eight domains of health status. The final score is standardised to the general population normative score of 50. The final score for each domain lies between 0 and 100, with 0 being the worst possible health and 100 the best possible health 
effects linear regression included older age at PAH diagnosis (coefficient $-0.1,95 \%$ CI 0.1 to $0.1, p=0.02$ ) while the presence of GIT manifestations (coefficient 0.7, 95\% CI -2.7 to $-0.1, p=0.04$ ) was associated with a worse SHAQ score over time. Neither PAH-specific therapy nor anticoagulation was associated with a significant change in the total SHAQ score after treatment (Table 4).

SSc-PAH patients had lower HRQoL scores across a number of domains of the SF-36 at PAH diagnosis, particularly in physical functioning, role-physical and general health and vitality, compared with the US normative mean of 50, indicating decreased HRQoL [21] and significantly lower SF-36 scores than SSc patients without PAH (Table 3). Determinants of worse SF-36 PCS using mixed effects linear regression included the presence of digital ulcers (coefficient $-3.7,95 \%$ CI -7.2 to

Table 4 Impact of PAH-specific therapy and anticoagulation on health-related quality of life scores in SSc-PAH determined through mixed effects linear regression modeling

\begin{tabular}{|c|c|c|}
\hline Variables & Coefficient $(95 \% \mathrm{Cl})$ & $P$ value \\
\hline \multicolumn{3}{|c|}{ Determinants of SF-36 physical component score } \\
\hline Female gender & $0.4(-3.9,4.7)$ & 0.85 \\
\hline Age at PAH diagnosis, years & $0.1(-0.1,0.2)$ & 0.29 \\
\hline Diffuse disease subtype & $-0.3(-3.8,3.2)$ & 0.87 \\
\hline Combination therapy & $1.7(-1.5,5.0)$ & 0.29 \\
\hline Anticoagulation therapy & $-3.7(-7.1,-0.3)$ & 0.03 \\
\hline GIT involvement ${ }^{\mathrm{a}}$ & $4.7(0.9,8.6)$ & 0.01 \\
\hline Digital ulcers ${ }^{a}$ & $-3.7(-7.2,-0.1)$ & 0.04 \\
\hline \multicolumn{3}{|c|}{ Determinants of SF-36 mental component score } \\
\hline Female gender & $-3.5(-8.6,1.6)$ & 0.18 \\
\hline Age at PAH diagnosis, years & $-0.1(-0.2,0.1)$ & 0.67 \\
\hline Diffuse disease subtype & $-1.1(-5.2,3.0)$ & 0.59 \\
\hline Combination therapy & $5.2(1.3,9.1)$ & 0.01 \\
\hline Anticoagulation therapy & $-2.5(-6.6,1.6)$ & 0.22 \\
\hline GIT involvement ${ }^{a}$ & $-1.6(-6.2,2.9)$ & 0.48 \\
\hline Digital ulcers ${ }^{a}$ & $2.8(-1.5,7.1)$ & 0.19 \\
\hline \multicolumn{3}{|l|}{ Determinants of SHAQ score } \\
\hline Female gender & $0.3(-0.5,0.9)$ & 0.46 \\
\hline Age at PAH diagnosis, years & $-0.1(-0.1,-0.1)$ & 0.02 \\
\hline Diffuse disease subtype & $-0.5(-1.1,0.2)$ & 0.13 \\
\hline Combination therapy & $-0.3(-0.8,0.3)$ & 0.38 \\
\hline Anticoagulation therapy & $0.3(-0.3,0.9)$ & 0.28 \\
\hline GIT involvement ${ }^{a}$ & $0.7(0.1,1.3)$ & 0.03 \\
\hline Digital ulcers ${ }^{\mathrm{a}}$ & $-0.1(-0.7,0.5)$ & 0.79 \\
\hline
\end{tabular}

Abbreviations: SSC systemic sclerosis, $P A H$ pulmonary arterial hypertension, GIT gastrointestinal involvement, SHAQ scleroderma health assessment questionnaire ${ }^{a}$ Disease manifestations present if present at PAH diagnosis or at any follow-up visit following $\mathrm{PAH}$ diagnosis
$-0.1, p=0.04$ ) and warfarin therapy (coefficient -3.7 , $95 \% \mathrm{CI}-7.1$ to $-0.3 ; p=0.03)$. The presence of GIT manifestations was associated with better SF-36 PCS score (coefficient 4.7, 95\% CI 0.9 to $8.6, p=0.01$ ). Combination therapy was not associated with a significant change in PCS scores after treatment (Table 4). Determinants of improved SF-36 MCS using mixed effects linear regression included treatment with combination PAH therapy (coefficient 5.2, 95\% CI 1.3-9.1, $p=0.01$ ) (Table 4).

\section{Discussion}

In our SSc-PAH cohort, the median overall survival was only 4 years with mortality of $45.5 \%$ over a follow-up period from study enrolment averaging 3.8 years. The one-year, two-year and three-year survival was $87.8 \%$, $78.3 \%$ and $61.7 \%$, respectively. Our results are similar to those in a recent French study (90\%, 78\% and 50\% survival) and lower than in a recent American study (93\%, $88 \%$ and $75 \%$ ), both of which also prospectively studied survival in a cohort with incident SSc-PAH [13, 22]. The majority of patients in the American study had New York Heart Association (NYHA) functional Class II disease at PAH diagnosis, which may account for the higher three-year survival in that study. Additionally, combination PAH therapy is more readily available in America than in Australia, which may partly explain the better survival in America. The NYHA functional class at PAH diagnosis was similar to ours in the French study. Mortality rates in the literature vary depending on whether cohorts include patients with incident disease only or a combination of those with incident and prevalent disease, with the potential of underreporting mortality in cohorts with prevalent disease due to survival bias. To our knowledge, this is the first paper to quantify YLL associated with SSc-PAH.

Of concern in our cohort, was that despite annual screening for $\mathrm{PAH}$, the majority of patients were in WHO functional Class III at PAH diagnosis. This may be because our screening algorithm missed patients with early or mild PAH without a markedly elevated RVSP, which may help to explain the relatively advanced stages of PAH observed in our study. It is becoming increasingly recognised that WHO functional class is an independent predictor of mortality [13, 23], as was shown in our cohort. The 6MWD was not associated with mortality in our study despite previous Australian data showing an association [11], suggesting that 6MWD is a nonspecific outcome measure for $\mathrm{PAH}$, and affected by the other complications of SSc.

Another independent predictor of mortality in our cohort included older age at $\mathrm{PAH}$ diagnosis, which has been reported in the literature to be a predictor of poor survival, with one study indicating that patients 
diagnosed with PAH over the age of 60 years had threefold higher mortality risk than those diagnosed under 60 years of age [22].

Certain clinical manifestations such as the presence of digital ulcers, calcinosis and telangiectasia have been reported to predict those patients at a higher risk of developing PAH [24-26]. In our study, the presence of digital ulcers was associated with greater mortality in SSc-PAH, which may represent a common underlying pathogenic mechanism involving endothelial dysfunction. Alternatively, it may be an indicator of recurrent infections or perhaps it identifies patients with a more severe vascular phenotype with obliterative vasculopathy involving the macrovasculature and microvasculature, manifesting in $\mathrm{PAH}$, digital ischaemia, ulcers and amputation.

The presence of moderate or severe ILD is in itself a risk factor for death in SSc $[27,28]$. In our cohort of patients, we excluded those with severe ILD defined as FVC $<60 \%$ and HRCT showing ILD, in whom PAH may have occurred secondary to ILD. However, we included patients with Group $1 \mathrm{PAH}$ and co-existent mild ILD defined by $\mathrm{FVC}>60 \%$ and mild abnormalities or no abnormalities on HRCT. Mild ILD was present in 51 patients (38.6\%) in our cohort and was predictive of death in SSc-PAH. We postulate that the co-existence of these two clinical manifestations could be due to shared underlying pathogenic mechanisms leading to a more severe clinical phenotype or that the occurrence in the lung of two independent pathologic conditions increases the risk of death.

There is evolving evidence to suggest that compared with monotherapy, the treatment of PAH with combination therapy is associated with improved survival in PAH. In small randomised trials and observational studies, combination therapy by means of "add-on" PAH therapy has consistently shown a survival benefit in PAH [11, 29, 30]. More recently, the treatment of PAH with upfront combination therapy compared with monotherapy showed not only a survival benefit, but also reduced hospitalisation for worsening $\mathrm{PAH}$ and disease progression [9].

Anticoagulation in PAH remains controversial despite some observational studies showing a survival benefit $[1,10]$. The survival benefit is particularly apparent in patients with iPAH as shown in the COMPERA study [31], Interestingly, this study did not show a survival benefit with anticoagulation in the non-idiopathic $\mathrm{PAH}$ group, which included patients with PAH secondary to CTD, congenital heart disease and portopulmonary hypertension. This may be due to the inclusion of these subgroups all within one category [31]. Furthermore, the REVEAL study showed no significant survival advantage in iPAH or SSc-PAH with the addition of anticoagulation [12]. Therefore, we believe that further research is required to assess the role of anticoagulation in PAH specifically associated with SSc.

Australian data collected between 2002 and 2009 identified a survival advantage with warfarin therapy in patients with CTD-PAH, the majority of whom had SSc [11]. In our study survival was similar if not worse than in patients diagnosed and treated between 2002 and 2009. Two reasons may explain this. First, our study included only patients with incident PAH whereas the previous study included both prevalent and incident cases, thus increasing potential survival bias. Second, survival in SSc-PAH is worse than that in CTD-PAH due to other autoimmune rheumatic diseases such as systemic lupus erythematosus and rheumatoid arthritis, so the overall survival may have been increased due to the inclusion of CTD-PAH "survivors".

There was improved survival in both studies with anticoagulation as an adjunct to PAH therapy compared with PAH therapy in isolation, despite patients on anticoagulation having had more severe $\mathrm{PAH}$ at baseline. We propose that the benefit of anticoagulation relates to the prevention of further micro-thrombotic phenomena occurring in the pulmonary vasculature, which likely plays an important role in the underlying pathogenesis of SSc$\mathrm{PAH}$. The results of our study provide a rationale for a randomised controlled trial evaluating anticoagulation as adjunct therapy in SSc-PAH, which the trial Systemic Sclerosis Pulmonary Hypertension Intervention with Apixaban (SPHInX) (ACTRN12614000418673) aims to resolve.

MMF has been shown to be associated with improved survival in small groups of patients with SSc-PAH [32]. We were not able to replicate these findings as we only had seven patients on MMF in our cohort, which may be explained by the limited availability and expense of MMF in Australia outside of tertiary hospitals until 2015. We were also interested in the relationship between the use of HCQ and survival in SSc-PAH given its anti-platelet effect. Our study identified a trend towards improved survival with HCQ, but this was not statistically significant. With only 12 patients on HCQ in our cohort, our study may not have been sufficiently powered to show such an association.

Not only does SSc-PAH affect survival, it also has a significant impact on patients' functional capacity and HRQoL $[15,16]$. Functional limitations, as captured by the SHAQ, were maintained over time with PAH therapy in our cohort, without a significant improvement in any specific domain, which is consistent with the literature $[33,34]$.

The physical component of HRQoL, as determined by the SF-36 PCS, was also not improved with combination $\mathrm{PAH}$ therapy in our cohort. However, combination PAH 
therapy was associated with a significant improvement in the mental component of HRQoL as measured using the SF-36 MCS. Reduction in mortality with minimal change in HRQoL has been previously reported in patients with CTD-PAH who are on PAH therapy [30, 34, 35]. The lack of improvement in the physical component of HRQoL following PAH treatment may reflect the complex, multifactorial and individual nature of HRQoL, which is impacted upon by a variety of factors that are difficult to measure and adjust for.

We recognise that there are limitations to our study. Lead-time bias may have contributed to the improvement in survival in patients diagnosed as a result of annual screening, with earlier implementation of PAHspecific therapy and anticoagulation. We excluded patients with co-existent PAH and severe ILD to ensure we captured only those patients with Group $1 \mathrm{PAH}$. Therefore we did not assess whether severe ILD contributed to mortality, although we assume it would, as mild ILD was a predictor of worse survival. In addition, treatment was not randomised; rather, it was prescribed at the individual physician's discretion as there is no standard nationwide protocol for the SSc-PAH treatment.

\section{Conclusion}

Despite advanced therapy, the median survival in SScPAH is only 4 years. In our study, the addition of anticoagulation to standard combination therapy was associated with a significant survival advantage, further pointing to mechanisms involving endothelial abnormalities and small vessel thrombosis in the pathogenesis of PAH. Although there was no significant improvement in physical function or in the physical components of HRQoL scores over time, these remained stable with PAH therapy.

\section{Additional file}

Additional file 1: Table S1. Patient characteristics by PAH status. Table S2. Baseline haemodynamics of SSC-PAH patients according to $\mathrm{PAH}$ therapy and anticoagulation. Table S3. Predictors of mortality in SSc-PAH determined by univariable analysis. Figure S1. Flowchart of SSC patient inclusion in the study. (DOC $85 \mathrm{~kb}$ )

\section{Abbreviations}

6MWD: Six-minute walk distance; ABS: Australian Bureau of Statistics: ANA: Anti-nuclear antibodies; APLA: Antiphospholipid antibodies; ASCS: Australian Scleroderma Cohort Study; ASIG: Australian Scleroderma Interest Group; CTD: Connective tissue disease; DLCO: Diffusing lung capacity for carbon monoxide; ENA: Extractable nuclear antigens; ERA: Endothelin receptor antagonists; FVC: Forced vital capacity; GAVE: Gastric antral vascular ectasia; HCQ: Hydroxychloroquine; HRCT: High-resolution computer tomography; HRQoL: Health-related quality of life; HRT: Hormone replacement therapy; ILD: Interstitial lung disease; INR: International normalised ratio; iPAH: Idiopathic pulmonary arterial hypertension; ICSSc: Limited cutaneous systemic sclerosis; Mci: mean cardiac index; MCS: Mental component scores; MMF: Mycophenolate mofetil; mPAP: Mean pulmonary arterial pressure; mRAP: Mean right atrial pressure; NYHA: New
York Heart Association; PAH: Pulmonary arterial hypertension PAWP: Pulmonary arterial wedge pressure; PBS: Pharmaceutical Benefit Scheme; PCS: Physical component scores; PDE5: Phosphodiesterase-5inhibitors; PFT: Pulmonary function test; PPIs: Proton pump inhibitors; PROs: Patient-reported outcomes; PVR: Pulmonary vascular resistance; RFTs: Respiratory function tests; RHC: Right heart catheterization; RVSP: Right ventricular systolic pressure; SF-36: Medical Outcomes Study Short Form-36; SHAQ: SSC-specific health assessment questionnaire; SMR: Standardised mortality ratio; SSc: Systemic sclerosis/scleroderma; TTE: Transthoracic echocardiogram; V/Q scan: Ventilation-perfusion scan; YLL: Years of life lost

\section{Acknowledgements}

Investigators of the Australian Scleroderma Interest Group (ASIG): we would like to acknowledge all investigators of the ASIG, including Catherine Hill, Adelaide, South Australia; Sue Lester, Adelaide, South Australia; Peter Nash, Sunshine Coast, Queensland; Gian Ngian, Melbourne Victoria; Mandana Nikpour, Melbourne, Victoria; Susanna Proudman, Adelaide, South Australia; Maureen Rischmueller, Adelaide, South Australia; Janet Roddy, Perth, Western Australia; Joanne Sahhar, Melbourne, Victoria; Wendy Stevens, Melbourne, Victoria; Gemma Strickland, Geelong, Victoria; Vivek Thakkar, New South Wales, Jenny Walker, Adelaide, South Australia; Jane Zochling, Hobart, Tasmania.

\section{Funding}

This work was supported by Scleroderma Australia, Arthritis Australia, Actelion Australia, Bayer, CSL Biotherapies, GlaxoSmithKline Australia and Pfizer. Dr Morrisroe holds an NHMRC Scholarship (APP1113954). Dr Nikpour holds an NHMRC Fellowship (APP1071735).

\section{Availability of data and materials}

All data generated or analysed during this study are included in this published article and its Additional files.

\section{Authors' contributions}

KM: study design, data analysis, interpretation of results and preparation of manuscript. WS: study design, data collection, interpretation of results and preparation of manuscript. $\mathrm{MH}$ : data analysis and preparation of manuscript. DP: data collection, interpretation of results and preparation of manuscript. JS: data collection, interpretation of results and preparation of manuscript. $\mathrm{GN}$ : data collection, interpretation of results and preparation of manuscript. DC: data collection, interpretation of results and preparation of manuscript. JZ: data collection, interpretation of results and preparation of manuscript. SP: data collection, interpretation of results and preparation of manuscript. MN: study design, data collection, data analysis, interpretation of results and preparation of manuscript. All authors read and approved the final manuscript.

\section{Competing interests}

The authors declare that they have no competing interests. SP, MN and WS have received grants from Actelion Pharmaceutical Auststralia, GlascoSmithKline and Scleorderma Australia. DP reports personal fees and non-financial support from Actelion Australia. The other authors declare that they have no competing interests.

\section{Ethics approval and consent to participate}

All patients provide written informed consent for collection of de-identified data, chart review and storage of serum and DNA for use in research studies including publications. All human research ethics committees of the participating sites have approved the ASCS (Royal Perth Hospital in Western Australia, Royal Adelaide and The Queen Elizabeth Hospitals in South Australia, Sunshine Coast Rheumatology and Prince Charles Hospital in Queensland, John Hunter, Royal Prince Alfred, Royal North Shore and St George Hospitals in New South Wales, Canberra Rheumatology in the Australian Capital territory, St Vincent's Hospital and Monash Medical Centre in Victoria and The Menzies Institute in Tasmania). Ethics Reference HREC-A 02/007.

\section{Publisher's Note}

Springer Nature remains neutral with regard to jurisdictional claims in published maps and institutional affiliations.

\section{Author details}

${ }^{1}$ Department of Medicine, The University of Melbourne at St Vincent's Hospital, 41 Victoria Parade, Fitzroy, 3065 Melbourne, Victoria, Australia. 
${ }^{2}$ Department of Rheumatology St Vincent's Hospital, 41 Victoria Parade, Fitzroy, 3065 Melbourne, Victoria, Australia. ${ }^{3}$ Monash University and Monash Health, 246 Clayton Road, Clayton 3168, Victoria, Australia. ${ }^{4}$ The University of Sydney at Royal Prince Alfred Hospital, Missenden Road, Camperdown 2050, NSW, Australia. ${ }^{5}$ Department of Rheumatology, Menzies Institute for Medical Research, Hobart, Australia. ${ }^{6}$ Rheumatology Unit, Royal Adelaide Hospital, North Terrace, Adelaide, SA 5000, Australia. ${ }^{7}$ Discipline of Medicine, University of Adelaide, Adelaide, SA 5000, Australia.

\section{Received: 10 February 2017 Accepted: 18 May 2017}

\section{Published online: 02 June 2017}

\section{References}

1. Chifflot H, Fautrel B, Sordet C, Chatelus E, Sibilia J. Incidence and Prevalence of Systemic Sclerosis: A systematic literature review. Semin Arthritis Rheum. 2008;37(4):223-35.

2. Hao Y, Hudson M, Baron M, Carreira P, Stevens W, Rabusa C, Tatibouet S, Carmona L, Joven BE, Huq M, et al. Early Mortality in a Multinational Systemic Sclerosis Inception Cohort. Arthritis Rheumatol. 2017;69(5):1067-77.

3. Tyndall AJ, Bannert B, Vonk M, Airo P, Cozzi F, Carreira PE, Bancel DF, Allanore $Y$, Muller-Ladner $U$, Distler $O$, et al. Causes and risk factors for death in systemic sclerosis: a study from the EULAR Scleroderma Trials and Research (EUSTAR) database. Ann Rheum Dis. 2010;69(10):1809-15.

4. Hao Y, Thakkar V, Stevens W, Morrisroe K, Prior D, Rabusa C, Youssef P, Gabbay E, Roddy J, Walker J, et al. A comparison of the predictive accuracy of three screening models for pulmonary arterial hypertension in systemic sclerosis. Arthritis Res Ther. 2015;17(1):7.

5. Muangchan C, Baron M, Pope J. The $15 \%$ rule in scleroderma: the frequency of severe organ complications in systemic sclerosis. A systematic review. J Rheumatol. 2013;40(9):1545-56.

6. D'Alonzo GE, Barst RJ, Ayres SM, Bergofsky EH, Brundage BH, Detre KM, Fishman AP, Goldring RM, Groves BM, Kernis JT, et al. Survival in patients with primary pulmonary hypertension. Results from a national prospective registry. Ann Intern Med. 1991;115(5):343-9.

7. Galie N, Humbert M, Vachiery JL, Gibbs S, Lang I, Torbicki A, Simonneau G, Peacock A, Vonk Noordegraaf A, Beghetti M, et al. 2015 ESC/ERS guidelines for the diagnosis and treatment of pulmonary hypertension. Revista espanola de cardiologia (English ed). 2016;69(2):177.

8. Barst RJ, Gibbs JS, Ghofrani HA, Hoeper MM, McLaughlin W, Rubin L, Sitbon O, Tapson VF, Galie N. Updated evidence-based treatment algorithm in pulmonary arterial hypertension. J Am Coll Cardiol. 2009;54(1 Suppl):S78-84.

9. Galie N, Barbera JA, Frost AE, Ghofrani HA, Hoeper MM, McLaughlin W, Peacock AJ, Simonneau G, Vachiery JL, Grunig $E$, et al. Initial use of ambrisentan plus tadalafil in pulmonary arterial hypertension. N Engl J Med. 2015;373(9):834-44.

10. Said K. Anticoagulation in pulmonary arterial hypertension: Contemporary data from COMPERA registry. Glob Cardiol Sci Pract. 2014;2014(2):48-52.

11. Ngian G, Nikpour M, Byron J, Tran A, Roddy J, Minson R, Hill C, Chow K, Sahhar J, Stevens W, et al. Survival in Australian patients with connective tissue disease-associated pulmonary arterial hypertension. Intern Med J. 2011:41:12.

12. Preston IR, Roberts KE, Miller DP, Sen GP, Selej M, Benton WW, Hill NS, Farber HW. Effect of warfarin treatment on survival of patients with pulmonary arterial hypertension (PAH) in the Registry to Evaluate Early and Long-Term PAH Disease Management (REVEAL). Circulation. 2015;132(25):2403-11.

13. Launay D, Sitbon O, Hachulla E, Mouthon L, Gressin V, Rottat L, Clerson P, Cordier JF, Simonneau G, Humbert M. Survival in systemic sclerosisassociated pulmonary arterial hypertension in the modern management era. Ann Rheum Dis. 2013;72(12):1940-6.

14. Thenappan T, Shah SJ, Rich S, Tian L, Archer SL, Gomberg-Maitland M. Survival in pulmonary arterial hypertension: a reappraisal of the NIH risk stratification equation. Eur Respir J. 2010;35(5):1079-87.

15. Shafazand S, Goldstein MK, Doyle RL, Hlatky MA, Gould MK. Health-related quality of life in patients with pulmonary arterial hypertension. Chest. 2004;126(5):1452-9.

16. Matura LA, McDonough A, Carroll DL. Health-related quality of life and psychological states in patients with pulmonary arterial hypertension. J Cardiovasc Nurs. 2014;29(2):178-84.

17. Preliminary criteria for the classification of systemic sclerosis (scleroderma). Subcommittee for scleroderma criteria of the American Rheumatism
Association Diagnostic and Therapeutic Criteria Committee. Arthritis Rheum 1980, 23(5):581-90

18. LeRoy EC, Medsger Jr TA. Criteria for the classification of early systemic sclerosis. J Rheumatol. 2001;28(7):1573-6.

19. Simonneau G, Gatzoulis MA, Adatia I, Celermajer D, Denton C, Ghofrani A, Gomez Sanchez MA, Kumar RK, Landzberg M, Machado RF, et al. Updated clinical classification of pulmonary hypertension. Turk Kardiyol Dern Ars. 2014;42 Suppl 1:45-54.

20. Johnson SR, Glaman DD, Schentag CT, Lee P. Quality of life and functional status in systemic sclerosis compared to other rheumatic diseases. J Rheumatol. 2006;33(6):1117-22.

21. Maglinte GA, Hays RD, Kaplan RM. US general population norms for telephone administration of the SF-36v2. J Clin Epidemiol. 2012;65(5):497-502.

22. Chung L, Domsic RT, Lingala B, Alkassab F, Bolster M, Csuka ME, Derk C, Fischer A, Frech T, Furst DE, et al. Survival and predictors of mortality in systemic sclerosis-associated pulmonary arterial hypertension: outcomes from the pulmonary hypertension assessment and recognition of outcomes in scleroderma registry. Arthritis Care Res (Hoboken). 2014;66(3):489-95.

23. Ngian GS, Stevens W, Prior D, Gabbay E, Roddy J, Tran A, Minson R, Hill C, Chow K, Sahhar J, et al. Predictors of mortality in connective tissue diseaseassociated pulmonary arterial hypertension: a cohort study. Arthritis Res Ther. 2012;14:R213.

24. Shah AA, Wigley FM, Hummers LK. Telangiectases in scleroderma: a potential clinical marker of pulmonary arterial hypertension. J Rheumatol. 2010;37(1):98-104.

25. Valenzuela A, Chung L. Calcinosis: pathophysiology and management. Curr Opin Rheumatol. 2015;27(6):542-8.

26. Karakulak UNHV, Maharjan N, Kaya EB, Kilic L, Akdoton A, Tokgozotlu L. Predictors of mortality in scleroderma patients with pulmonary hypertension. J Am Coll Cardiol. 2013;62(14):C17.

27. Bussone G, Mouthon L. Interstitial lung disease in systemic sclerosis. Autoimmun Rev. 2011;10(5):248-55.

28. Mathai SC, Hummers LK, Champion HC, Wigley FM, Zaiman A, Hassoun PM, Girgis RE. Survival in pulmonary hypertension associated with the scleroderma spectrum of diseases: impact of interstitial lung disease. Arthritis Rheum. 2009;60(2):569-77.

29. Simonneau G, Rubin LJ, Galie N, Barst RJ, Fleming TR, Frost AE, Engel PJ, Kramer MR, Burgess G, Collings L, et al. Addition of sildenafil to long-term intravenous epoprostenol therapy in patients with pulmonary arterial hypertension: a randomized trial. Ann Intern Med. 2008;149(8):521-30.

30. Ghofrani H-A, Galiè N, Grimminger F, Grünig E, Humbert M, Jing Z-C, Keogh AM, Langleben D, Kilama MO, Fritsch A, et al. Riociguat for the treatment of pulmonary arterial hypertension. N Engl J Med. 2013;369(4):330-40.

31. Olsson KM, Delcroix M, Ghofrani HA, Tiede H, Huscher D, Speich R, Grunig E, Staehler G, Rosenkranz S, Halank M, et al. Anticoagulation and survival in pulmonary arterial hypertension: results from the Comparative, Prospective Registry of Newly Initiated Therapies for Pulmonary Hypertension (COMPERA). Circulation. 2014;129(1):57-65.

32. Virginia S, Aryeh F. Mycophenolate Mofetil Use In Pulmonary Hypertension In Systemic Sclerosis. Observations From The PHAROS Cohort. In: C54 treatment of pulmonary hypertension. Am J Respir Crit Care Med. 2014;189: A4790.

33. Kowal-Bielecka O, Avouac J, Pittrow D, Huscher D, Behrens F, Denton CP, Foeldvari I, Humbert M, Matucci-Cerinic M, Nash P, et al. Analysis of the validation status of quality of life and functional disability measures in pulmonary arterial hypertension related to systemic sclerosis: results of a systematic literature analysis by the expert panel on outcomes measures in pulmonary arterial hypertension related to systemic sclerosis (EPOSS). J Rheumatol. 2011;38(11):2419-27.

34. Denton CP, Pope JE, Peter HH, Gabrielli A, Boonstra A, van den Hoogen FH, Riemekasten G, De Vita S, Morganti A, Dolberg M, et al. Long-term effects of bosentan on quality of life, survival, safety and tolerability in pulmonary arterial hypertension related to connective tissue diseases. Ann Rheum Dis. 2008;67(9):1222-8

35. Taichman DB, Shin J, Hud L, Archer-Chicko C, Kaplan S, Sager JS, Gallop R, Christie J, Hansen-Flaschen J, Palevsky H. Health-related quality of life in patients with pulmonary arterial hypertension. Respir Res. 2005;6(1):92. 